\title{
Bioanalysis
}

\section{The importance of optimal extraction to insure the reliable MS-based assays of endogenous compounds}

\author{
"The endogenous compounds include large biomolecules such as proteins and DNA \\ and small molecules such as steroids."
}

Keywords: endogenous $\bullet$ LC-MS/MS • optimal extraction • progesterone

Endogenous compounds are naturally occurring compounds within an organism, tissue or cell. The endogenous compounds include large biomolecules such as proteins and DNA and small molecules such as steroids. The present discussion will cover the small molecules in the circulation (plasma, serum and blood) due to the fundamental difference between biomolecules and circulating small molecules.

In order to measure endogenous compounds with precision and accuracy, the biosamples usually need to be cleaned up. Accordingly, the calibration curves for endogenous compounds are usually prepared in stripped serum or surrogate matrices. Liquid-liquid extraction (LLE) is a widely used for biosample cleanup by reducing the matrix effect while maintaining a good sensitivity $[1,2]$. The basic principle is that target analyte partitions from the aqueous phase (i.e., serum, plasma) into the organic phase which has a good solubility for analytes. The partitioning efficiency of the analytes from the aqueous into the organic phase is determined by the polarity of both the organic solvent and the target analyte, in other words, solvent polarity and miscibility, or the solubility of the analyte in the solvent. However, in addition to the target analytes, many other 'invisible' compounds can be co-extracted with the target analytes from serum or plasma. For instance, unconjugated steroids are endogenous compounds which can be easily interfered with by many unknown natural compounds present in the biological matrix and even in the environment if the extraction solvent is not adequately chosen.
For example, the noise level at LC-MS/ MS analysis of the progesterone, with the detection mode of multiple reaction monitoring (MRM), and the number and intensity of the potential interference peaks in unstripped serum increases significantly with the polarity of the extraction solvent going from hexanes, 1-chlorobutane (Cl-butane), methyl tert-butyl ether (MTBE)/hexanes (1:1 in volume), MTBE to ethyl acetate (EtOAc)/hexanes (1:1 in volume), [3] where the indexes are $0,1,1.25,2.5,2.2$, respectively [4]. Due to the lack of data, the index of MTBE/hexanes and EtOAc/hexanes is the simple average of two solvents. By contrast, in stripped serum, none of these solvents gives a high level of noise. This observation is attributed to the stripping process where many potential interferences with steroids have been removed. Usually, the absorption of serum by activated charcoal will remove the steroidal compounds and other small molecules, while serum proteins remain [5].

\section{Can the different MRM transitions resolve the analyte from potential interferences?}

MRM quantitation mode has high specificity since it only counts signals if and only if both the parent ion and product ion match those in the preset MRM transition. This detection mode is popular and shows high specificity for drug analysis in biological matrices. Accordingly, LC-MS/MS has become the standard quantitation technique for the quantitation of compounds in the pharmaceutical, food and environment industries.

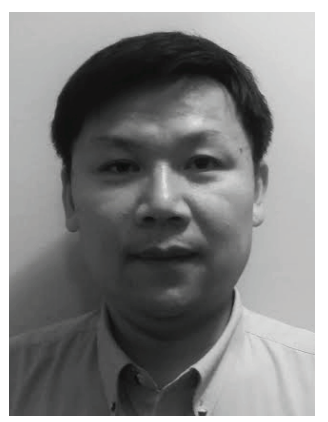

Yuyong Ke EndoCeutics Inc., 2795 Laurier Blvd, Suite 500, Quebec (QC) G1V 4M7, Canada

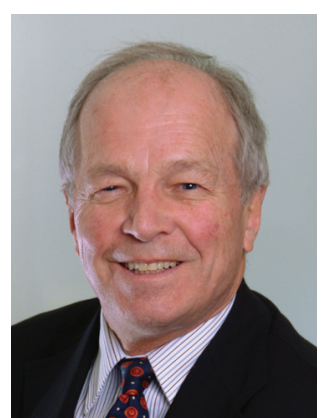

Fernand Labrie Author for correspondence: EndoCeutics Inc., 2795 Laurier Blvd, Suite 500, Quebec (QC) G1V 4M7, Canada Tel.: +1 4186530055 fernand.labrie@endoceutics.com 
Naturally occurring endogenous compounds, especially compounds with small molecular weight are easily interfered by unknown compounds from biological matrix and environmental impurities. Usually, in order to ensure the reliability of the method, the ion ratio of two MRM transitions can be used to verify the analyte purity in unknown samples by comparing that ratio obtained with that of the standards. A reasonable acceptance criterion for the difference of ion ratio between unknowns and standards is within $15 \%[6,7]$. A deviation of the ratio out of the acceptance criteria range indicates that the peak contains unknown interferences, resulting in inaccurate data. Unfortunately, the ratio cannot tell which transition can give you reliable data. Instead, a positive or negative deviation can tell which transition is better than the other for this particular sample.

\section{"Naturally occurring endogenous compounds, especially compounds with small molecular weight are easily interfered by unknown compounds from biological matrix and environmental impurities."}

Using MTBE/hexanes (1:1 in volume) as extraction solvents, the ion ratios of two MRM transitions observed in many unknown serum samples when monitoring progesterone are out of the acceptance criteria range while when MTBE or more polar solvents are used as extraction solvents, the ion ratios of all unknown samples are out of the acceptance criterion range [3].

\section{Can a long gradient time resolve the analyte from potential interferences?}

Usually, the selection of different columns and the choice of different gradients offer solution to resolve the analyte from interferences. These strategies are usually very effective in resolving analytes from known interferences such as changing the gradient, even the mobile phase, and trying different columns.

For unknown interferences, it is somewhat like a black box. Due to the lack of standards and structure information of the interferences as well as the number of interferences, it becomes a complex task. Some interferences can be separated by changing the gradient or using different columns while other interferences peak may then merge with that of the analyte. With the example of progesterone, the retention time of progesterone changes from $5.5 \mathrm{~min}$ to $10.2 \mathrm{~min}$ by changing the gradient [3]. Although the progesterone is well resolved visually, the ion ratio and quantitation data of unknowns clearly show that a longer run time, in other words, less organic in mobile phase does not really benefit the separation of progesterone from interferences. Unfortunately, the values of progesterone measured with longer run time are even slightly higher than those with short run time following the polar solvent extraction.

\section{Can supported liquid extraction resolve the analyte from the potential interferences?}

Compared with traditional LLE, supported liquid extraction (SLE) is ready for automation by reducing manual labor. Can SLE simply resolve the issue encountered with LLE? The answer is no from the experience of progesterone. First, SLE from different vendors shows different performance [Ke Y, Labrie F, Unpublished Data]. Second many unknown interferences could be co-eluted with the analyte. This is similar to the observations with LLE. In addition, surface absorption of SLE could bring the other potential interferences, similar to the case of the glass tube used for LLE. Sometimes, the prewash of SLE may be necessary similar to other solid phase extractions (SPEs).

\section{Strategies for optimal extraction of samples}

First, if there are two or more MRM transitions available for the analyte, the ion ratio from two different MRM transitions should be used to monitor the peak purity during method development for all the QCs, calibrators and unknowns. The criterion is that the ion ratio should be close enough (recommendation is $<15 \%$ ) from the unknown sample to the standard. Second, with the setup and acceptance criteria of the ion ratio, the sample preparation should be optimized. For the LLE, SLE or SPE, extraction solvents or eluents with different polarity should be tried in order to choose optimal extraction solvent or eluent, thus to have the quantification results (ion ratio) of unknown samples unchanged with different MRM transitions or different gradients, mobile phases and columns. The recovery during extraction should also be taken into consideration in order to perform an optimal extraction. If there is only one transition available for the analyte, besides the optimal extraction, additional work such as different columns, different mobile phases and different gradients should be tried to ensure the reliable data.

\section{The optimization example of liquid-liquid extraction: progesterone}

With the above-mentioned strategies, two progesterone MRM transitions of 315>97 and 315>109 are used for the ion ratio while a series of extraction solvents, namely hexanes, Cl-butane, MTBE/hexanes (1:1), MTBE and EtOAc/hexanes (1:1) have been used [3]. The progesterone value from the Cl-butane extraction is identical 
to that using the hexanes extraction with either transition although the polarity index of Cl-butane (1) is significantly different from that of hexanes $(0)$, which are not affected by different MRM transitions or different gradients. With MTBE/hexanes (1:1 in volume), the rough polarity index of 1.25 makes the accuracy of data questionable. This may indicate that a threshold of polarity index exists for obtaining clean progesterone samples, in other words, an optimal value of polarity index range (0-1) of extraction solvent could be appropriate for the accurate assay of endogenous progesterone [8]. With this optimal extraction, the progesterone concentration measured in postmenopausal serum is in the range of $5.74-91.7 \mathrm{pg} / \mathrm{ml}$, which is much lower than the reported range of $314-942 \mathrm{pg} / \mathrm{ml}$ using immuoassays [9].

\section{Summary}

Specificity is a challenge for accurate determination of the concentration of endogenous compounds in biological matrices which can be affected by the sample preparation and the choice of the MRM transition of

\section{References}

1 Penning TM, Lee SH, Jin Y, Gutierrez A, Blair IA. Liquid chromatography-mass spectrometry (LC-MS) of steroid hormone metabolites and its applications. J. Steroid Biochem. Mol. Biol. 121(3-5), 546-555 (2010).

2 Ke Y, Bertin J, Gonthier R, Simard JN, Labrie F. A Sensitive, simple and robust LC-MS/MS method for the simultaneous quantification of seven androgen- and estrogen-related steroids in postmenopausal serum. J. Steroid Biochem. Mol. Biol. 144, 523-534 (2014).

3 Ke Y, Labrie F. Impact of serum sample extraction on the accurate measurement of progesterone by liquid chromatography tandem mass spectrometry: an example of importance of extraction of endogenous compounds. Presented at: 9th Workshop on Recent Issues in Bioanalysis. Miami, FL, USA, 13-17 April (2015).

4 Snyder LR. Classification of the solvent properties of common liquids. J. Chrom. A 92(22), 223-230 (1974).

5 Carter P. Preparation of ligand-free human serum for radioimmunoassay by adsorption on activated charcoal. Clin. Chem. 24(2), 362-364 (1978). the LC-MS/MS. Therefore, the sample preparation procedure should be optimized for each endogenous compound. In the meantime, during the method development stage, the quantitation should be compared or the ion ratio of two MRM transitions should be monitored, not only with qualifiers. The optimal extraction not only reduces the baseline but also limits the potential interferences. Monitoring of the ion ratio or quantitation using two different MRM transitions can ensure the specificity, thus leading to a more reliable measurement of endogenous compounds. These strategies are very important for the accurate quantitation of endogenous compounds either with LLE or SPE.

\section{Financial \& competing interests disclosure}

The research was supported by Endoceutics. The authors have no other relevant affiliations or financial involvement with any organization or entity with a financial interest in or financial conflict with the subject matter or materials discussed in the manuscript apart from those disclosed.

No writing assistance was utilized in the production of this manuscript.

6

Guidance for Industry. Bioanalytical Method Validation -

Revision 1. U.S. Department of Health and Human Services, Food and Drug Administration, Center for Drug Evaluation and Research (CDER), Center for Veterinary Medicine (CVM). Division of Drug Information, WO51, Room 2201, Center for Drug Evaluation and Research, Food and Drug Administration. www.fda.gov

7 Rauh M, Groschl M, Rascher W, Dorr HG. Automated, fast and sensitive quantification of 17-hydroxy-progesterone, androstenedione and testosterone by tandem mass spectrometry with on-line extraction. Steroids 71, 450-458, (2006).

8 Kinoshita T, Honma S, Shibata Y et al. An innovative LCMS/MS-based method for determining CYP 17 and CYP 19 activity in the adipose tissue of pre- and postmenopausal and ovariectomized women using 13C-labeled steroid substrates. J. Clin. Endocrinol. Metab. 99(4), 1339-1347 (2014).

9 Schumacher M, Mattern C, Ghoumari A et al. Revisiting the roles of progesterone and allopregnanolone in the nervous system: resurgence of the progesterone receptors. Prog. Neurobiol. 113, 6-39 (2014). 